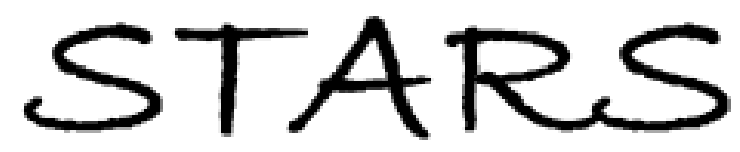

University of Central Florida

STARS

$1-1-2013$

\title{
Droplets on liquid surfaces: Dual equilibrium states and their energy barrier
}

\author{
Roxana Shabani \\ University of Central Florida \\ Ranganathan Kumar \\ University of Central Florida \\ Hyoung J. Cho \\ University of Central Florida
}

Find similar works at: https://stars.library.ucf.edu/facultybib2010 University of Central Florida Libraries http://library.ucf.edu

This Article is brought to you for free and open access by the Faculty Bibliography at STARS. It has been accepted for inclusion in Faculty Bibliography 2010 s by an authorized administrator of STARS. For more information, please contact STARS@ucf.edu.

\section{Recommended Citation}

Shabani, Roxana; Kumar, Ranganathan; and Cho, Hyoung J., "Droplets on liquid surfaces: Dual equilibrium states and their energy barrier" (2013). Faculty Bibliography 2010s. 4679.

https://stars.library.ucf.edu/facultybib2010/4679

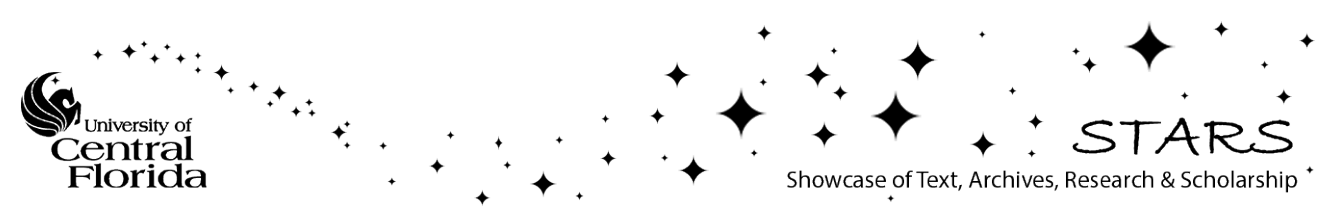




\section{Droplets on liquid surfaces: Dual equilibrium states and their energy barrier}

Cite as: Appl. Phys. Lett. 102, 184101 (2013); https://doi.org/10.1063/1.4804242

Submitted: 12 March 2013 . Accepted: 21 April 2013 . Published Online: 07 May 2013

Roxana Shabani, Ranganathan Kumar, and Hyoung J. Cho
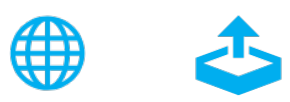

\section{ARTICLES YOU MAY BE INTERESTED IN}

Droplet actuation on a liquid layer due to thermocapillary motion: Shape effect

Applied Physics Letters 96, 264101 (2010); https://doi.org/10.1063/1.3456391

Drop coalescence through a liquid/liquid interface

Physics of Fluids 16, 2170 (2004); https://doi.org/10.1063/1.1735686

Electrowetting-based actuation of liquid droplets for microfluidic applications

Applied Physics Letters 77, 1725 (2000); https://doi.org/10.1063/1.1308534

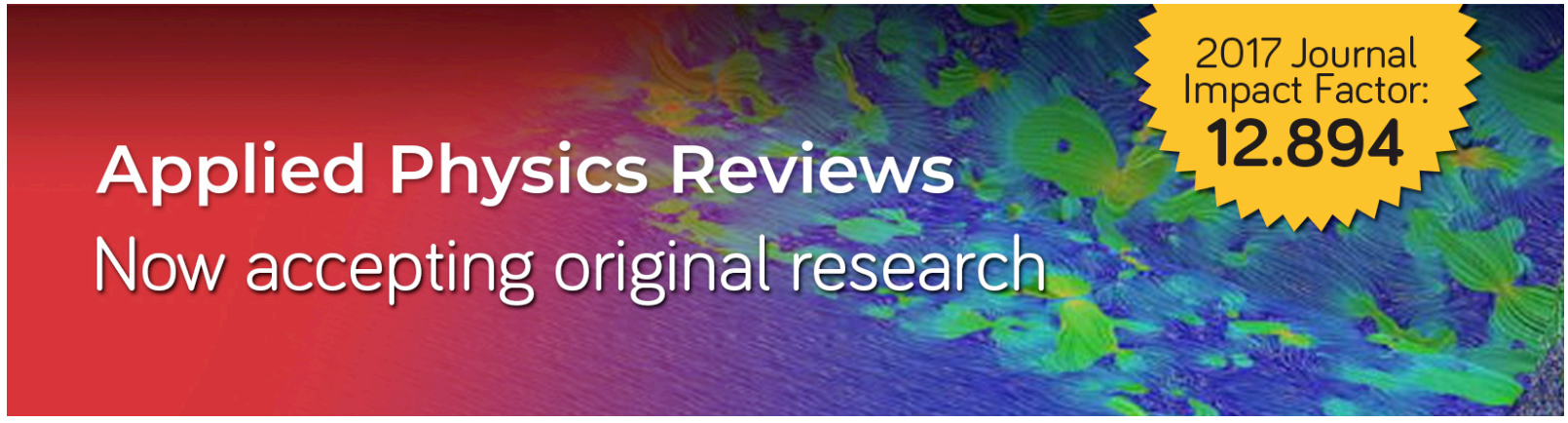




\title{
Droplets on liquid surfaces: Dual equilibrium states and their energy barrier
}

\author{
Roxana Shabani, ${ }^{1}$ Ranganathan Kumar, ${ }^{1}$ and Hyoung J. Cho ${ }^{1,2, a)}$ \\ ${ }^{1}$ Department of Mechanical and Aerospace Engineering, University of Central Florida - Orlando, \\ Florida 32816-2450, USA \\ ${ }^{2}$ School of Advanced Materials Science and Engineering, Sungkyunkwan University, Suwon 440-746, \\ South Korea
}

(Received 12 March 2013; accepted 21 April 2013; published online 7 May 2013)

\begin{abstract}
Floating aqueous droplets were formed at oil-air interface, and two stable configurations of (i) non-coalescent droplet and (ii) cap/bead droplet were observed. General solutions for energy and force analysis were obtained for both configurations and were shown to be in good agreement with the experimental observations. The energy barrier obtained for transition from configuration (i) to configuration (ii) was correlated to the droplet release height and the probability of non-coalescent droplet formation. @ 2013 AIP Publishing LLC. [http://dx.doi.org/10.1063/1.4804242]
\end{abstract}

The formation of floating aqueous droplets on the free surface of immiscible liquids has application in dropletbased microfluidic devices. ${ }^{1-3}$ The floating aqueous droplets could be used as containers for encapsulating reagents in biochemical reactions. ${ }^{4}$ They allow low consumption of the reagents and give direct access to reaction products. ${ }^{4}$ The non-coalescent (NC) droplets have been formed by surface tension gradient due to a temperature difference between the droplet and the pool liquid, ${ }^{3}$ vertical oscillation of base liquid, ${ }^{5}$ and coating droplets with non-wetting hydrophobic powder. ${ }^{6} \mathrm{NC}$ droplets formation on a liquid interface in isothermal condition has also been reported. ${ }^{7}$ Although the floating droplet has been of interest, a general solution has not been provided for the dual equilibrium states. ${ }^{3,7,8}$ Further, while the non-coalescence of a droplet on the free surface of the same liquid is widely studied, the studies using immiscible liquids are scarce. In this work, an oil phase (FC-43, 3M) at $25^{\circ} \mathrm{C}$ with a surface tension, $\gamma_{\text {oa }}$, of $16 \mathrm{mN} / \mathrm{m}$ and a density, $\rho_{\mathrm{o}}$, of $1860 \mathrm{~kg} / \mathrm{m}^{3}$ was used as a base medium for formation of floating droplets. A droplet of water at room temperature $\left(25^{\circ} \mathrm{C}\right)$ with a density, $\rho_{\mathrm{w}}$, of $997 \mathrm{~kg} / \mathrm{m}^{3}$ was released on the free oil surface using a high precision needle connected to a syringe pump. The water droplets are released due to a gravity driven pinch-off from the tip of the needle. ${ }^{8}$ The stable configuration of the droplet at the air-oil interface depends on the height from which the droplet is released and the surface tension of the three phases. If the droplet is released from within a range of height, it will not rupture the free surface as it does not have sufficient momentum. ${ }^{7}$ Instead, it will rest on the stretched free surface which is deformed by the weight of the NC droplet (Figs. 1(a) and $1(\mathrm{c})$ ). Bond number (defined as $\mathrm{g} \rho_{\mathrm{w}} \mathrm{R}_{\mathrm{D}}{ }^{2} / \gamma_{\text {oa }}$ ) for a droplet radius, $R_{D}$, with an order of $1 \mathrm{~mm}$ is small $(\sim 0.1)$, and hence a uniform pressure is assumed inside the droplet. The droplet is assumed to be spherical from Laplace pressure equation. ${ }^{3}$ By increasing the droplet release height, $\mathrm{H}_{\mathrm{R}}$, it gains more energy to rupture the free surface and becomes submerged. However the droplet is held up by the buoyancy force $\left(\rho_{\mathrm{o}}>\rho_{\mathrm{w}}\right)$ and becomes stable at the oil-air interface

\footnotetext{
${ }^{\text {a) }}$ Author to whom correspondence should be addressed. Electronic mail: hjcho@ucf.edu
}

(Figs. 1(b) and 1(d)). These water droplets have two parts of cap in air and bead in oil (C/B droplet). A triple contact line (TCL) is formed at the circular perimeter of the intersection of the spherical cap and bead.

The probability of formation of NC droplets was studied as a function of the droplet's $H_{R}$. Different gauge needles $(35 \mathrm{G}-23 \mathrm{G})$ were used to release droplets of different sizes. A hundred droplets were released from each needle and weighed to calculate the equivalent $R_{D}$ for each needle. ${ }^{9}$ The probability of formation of the $\mathrm{NC}$ droplets (percentage of the observed NC droplets to the total droplets released) is measured as a function of $H_{R}$ for different $R_{D}$. Fig. 2 shows the results as a function of Weber number which is the ratio of the droplet kinetic energy to the base medium surface energy $\left(4 \rho_{\mathrm{w}} \mathrm{g} \mathrm{H}_{\mathrm{R}} \mathrm{R}_{\mathrm{D}}\right) / \gamma_{\mathrm{oa}}$. It could be used to show whether the released droplet from a specific $\mathrm{H}_{\mathrm{R}}$ has sufficient kinetic momentum to overcome the liquid surface energy and break up its free surface. As Weber number increases, breaking up the free surface becomes easier for the droplets, and the probability of formation of $\mathrm{NC}$ droplets becomes zero. However, the complexity of $\mathrm{NC}$ droplet formation could not be captured by We number only. Fig. 2 shows that for a similar Weber number the probability increases as $R_{D}$ decreases. This is due to the fact that Weber number compares the droplet kinetic energy with the surface energy of the base medium, which is not the only obstacle that a falling droplet faces to break the surface. Other energy terms such as the gravitational potential energy of the displaced base medium and energy used for wave formation should be taken into account as discussed in the next section. For each droplet size there is a transition region, in which the probability of forming NC droplets changes from $100 \%$ to $0 \%$ by increasing $H_{R}$ (Fig. 2). This transition region is wider for higher $R_{D}$ and becomes sharper as $R_{D}$ decreases. $\mathrm{NC}$ droplet formation in transition region shows a statistical nature which is more pronounced for larger droplets. This might be understood in terms of wave energy dissipation and the time required for damping the initial oscillation of droplet surface upon pinching off from the needle. While a small droplet quickly attains its stable shape (spherical) the lager droplet may not have enough time to dissipate the facial waves which may affect the $\mathrm{NC}$ to $\mathrm{C} / \mathrm{B}$ transition. 


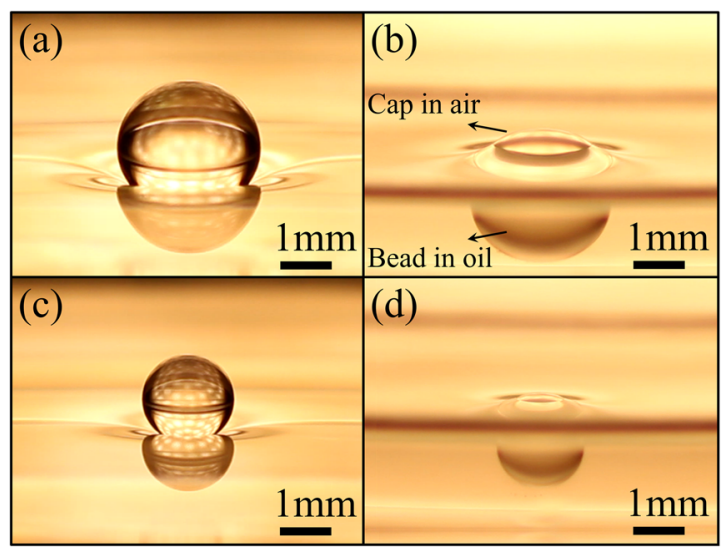

FIG. 1. Stable configurations of aqueous droplets at oil-air interface: (a) NC droplet resting on a stretched and deformed free surface. (b) C/B droplet with TCL. (c), (d) The effect of the droplet size on the deformation of the free surfaces.

Even the droplets that survive the first impact and stay on the surface (NC droplets) will at some point coalesce with the oil surface and sink down to C/B configuration. ${ }^{9,10}$ The latter has a lower energy and thus is more stable while the $\mathrm{NC}$ configuration is metastable. Several studies have focused on the physical origin of $\mathrm{NC}$ droplets. ${ }^{3,5,6,9-12}$ A thin film of air between the droplet and the free surface is believed to be responsible for the formation of $\mathrm{NC}$ droplets by preventing the two liquids to wet. ${ }^{9,11,13-15}$ The existence of such film has been reported by observing the mirror-like reflection, an infinite electrical resistance,${ }^{16}$ and no occurrence of material transfer ${ }^{9}$ between the droplet and the base medium. The air layer thickness was reported to be about $10 \mu \mathrm{m},{ }^{3,16}$ much larger than the van der Waals attraction range $(10 \mathrm{~nm}){ }^{9}$ The air film's slow drainage postpones droplet coalescence ${ }^{13}$ due to the lift generated by the lubrication effect of the flowing air film. ${ }^{5}$

Two different sets of parameters were defined to describe the geometries of the two observed configurations (Figs. 3(a) and 3(b)). The ratio of the radii of curvature of the cap and bead ( $R_{\text {cap }}$ and $R_{\text {bead }}$, respectively) is proportional to the ratio of surface tension of water in contact with air and oil ( $\gamma_{\mathrm{wa}}$ and $\gamma_{\mathrm{wo}}$, respectively) due to uniform Laplace pressure in the droplet. ${ }^{1} \gamma_{\mathrm{wa}}$ and $\gamma_{\mathrm{oa}}$ are known to be

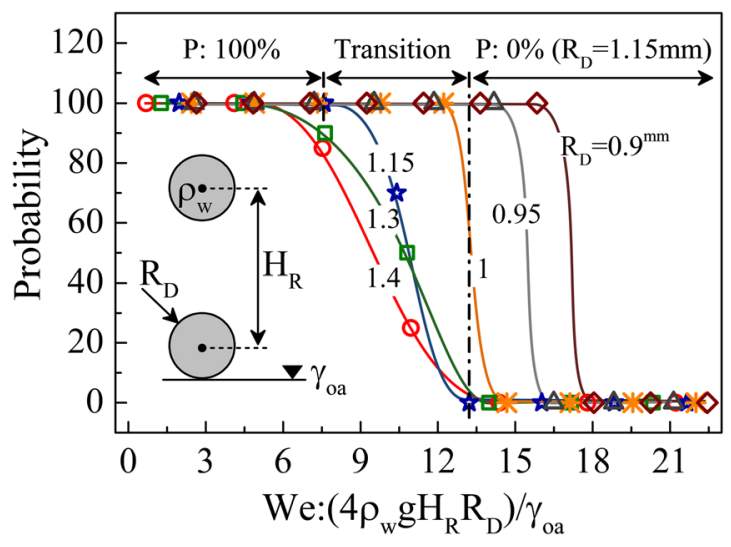

FIG. 2. The probability of formation of NC droplets versus Weber number obtained for different droplet radii, $\mathrm{R}_{\mathrm{D}}$.

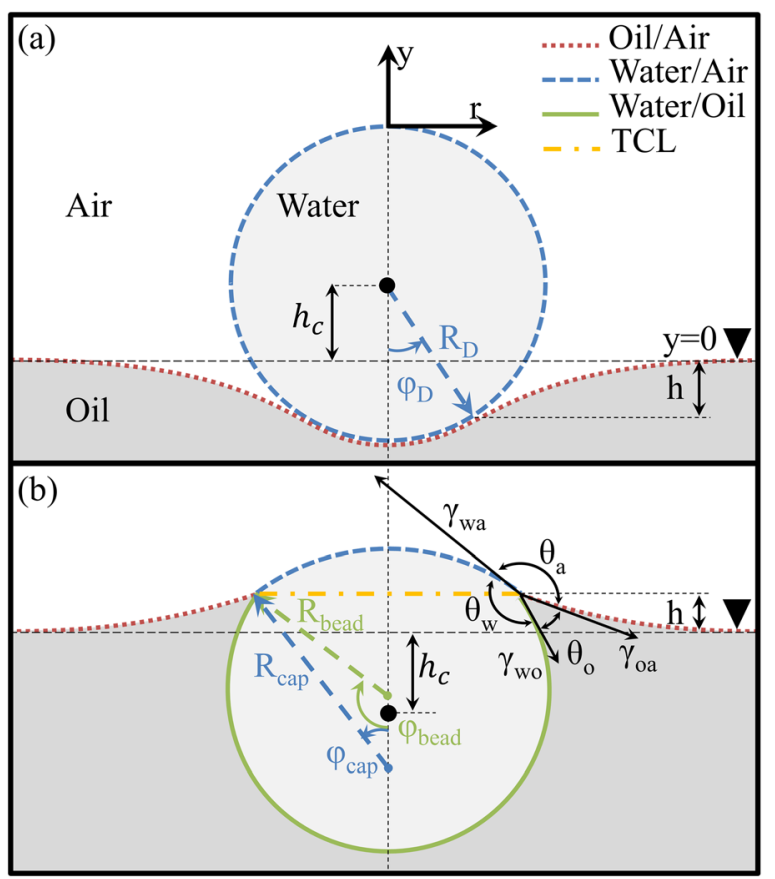

FIG. 3. Physical and geometrical parameters used in energy analysis of (a) $\mathrm{NC}$ droplet and (b) C/B droplet configurations.

$72 \mathrm{mN} / \mathrm{m}$ and $16 \mathrm{mN} / \mathrm{m}$, respectively, at $25^{\circ} \mathrm{C}$. The interfacial tension between water and FC-43, $\gamma_{\text {wo }}$, was estimated to be $56.5 \pm 0.5 \mathrm{mN} / \mathrm{m}$ using a method shown by Fox. ${ }^{17}$ The three pairs of interfacial tension satisfy the Neumann's inequalities, which means none of the interfacial tension between any pair of fluids exceed the sum of the other two. ${ }^{18}$

The radius of the cap's circular base area, $\mathrm{R}_{\text {cap }} \sin \left(\varphi_{\text {cap }}\right)$, is equal to the radius of the bead's base area, $R_{\text {bead }}$ $\sin \left(\varphi_{\text {bead }}\right)$, where $\varphi_{\text {cap }}$ and $\varphi_{\text {bead }}$ are defined in Fig. 3(b). The droplet volume is constant and is equal to the sum of the cap and the bead volumes. The net force per unit length of TCL has to be zero in a stable configuration, and therefore knowing the three surface energies and using Neumann's triangle concept, three contact angles of $\theta_{\mathrm{a}}, \theta_{\mathrm{o}}$, and $\theta_{\mathrm{w}}$ (defined inside air, oil, and water, respectively) are found.

The total energy of both floating droplet configurations, $E_{\text {net }}$, is the sum of the gravitational potential energies of (i) the water droplet, $\mathrm{U}_{\mathrm{D}}$, and (ii) the displaced liquid volume of the base medium, $U_{D L}$, and (iii) the total of the surface energies of the interfaces between each two phases, $\mathrm{E}_{\mathrm{S}}$,

$$
E_{\text {net }}=U_{D}+U_{D L}+E_{S},
$$

The droplet separation angle, $\varphi_{\mathrm{D}}$, is defined in Fig. 3(a) (also see Fig. S1(a)). ${ }^{19} \varphi_{\mathrm{D}}$ for the NC droplet and $\varphi_{\text {cap }}$ for the $\mathrm{C} / \mathrm{B}$ droplet were changed as the independent variables. The droplet vertical position varies as $\varphi_{\mathrm{D}}$ for $\mathrm{NC}$ droplet ( $\varphi_{\text {cap }}$ for $\mathrm{C} / \mathrm{B}$ droplet) changes. Assuming that the pool of the base medium is large, the Young-Laplace equation for an axisymmetric problem (in the cylindrical coordinates) was used to describe the free surface profiles of the base medium, in both configurations. ${ }^{3,8,18,20}$ The Young-Laplace equation is a relation between the hydrostatic pressure difference across the oil-air interface and its local mean curvature 


$$
\begin{aligned}
\rho_{o} g y= & \gamma_{o a}\left(\left(d^{2} y / d r^{2}\right) /\left(1+(d y / d r)^{2}\right)^{3 / 2}\right. \\
& \left.+(d y / d r) /\left(r\left(1+(d y / d r)^{2}\right)^{1 / 2}\right)\right)
\end{aligned}
$$

where $y$ and $r$ are the height and the radial distance of a point on the free surface profile in cylindrical coordinates (Figs. 3(a) and 3(b)). The profile of the free surface, the area of the deformed oil-air interface, the volume of the displaced base medium, and finally $\mathrm{E}_{\text {net }}$ are calculated numerically (Figs. S1(a) and S1(b)). ${ }^{19}$ The energy analysis predicted a minimum of $E_{n e t}$ (equilibrium state) for each configuration. Using numerical values, three-dimensional images of a floating droplet were generated at the oil-air interface in both equilibrium configurations. These images depicted in Fig. 4 show good agreement with the pictures taken from floating droplets with similar sizes, for both configurations in Fig. 1.

Smaller droplets deform the surface to a less extent as compared to larger ones (Figs. 4(c) and 4(d)). $\varphi_{\mathrm{D}}$ for $\mathrm{NC}$ droplets and $\varphi_{\text {cap }}$ for $\mathrm{C} / \mathrm{B}$ droplets were measured for $\mathrm{R}_{\mathrm{D}}$ of $0.9 \mathrm{~mm}-1.4 \mathrm{~mm}$ (needle gauges of $35 \mathrm{G}-23 \mathrm{G}$ ). A good agreement between the experimental data and the values predicted by energy analysis were obtained (Fig. 4(e)). The gravity contribution as compared to that of the surface tension force becomes negligible at small droplet size limit $\left(R_{D} \rightarrow 0\right)$, due to the scaling effect. Therefore the energy analysis results in $\varphi_{\mathrm{D}}=0$ for a $\mathrm{NC}$ droplet but a non-zero $\varphi_{\text {cap }}$ for a $\mathrm{C} / \mathrm{B}$ droplet $\left(\pi-\theta_{\mathrm{a}}\right)$ when $\mathrm{R}_{\mathrm{D}}$ approaches zero.

To further confirm the results obtained by energy analysis, force analysis was also conducted (Figs. S2(a) and S2(b)). ${ }^{19,21,22}$ A perfect agreement was obtained between the two methods, and similar values for equilibrium states were

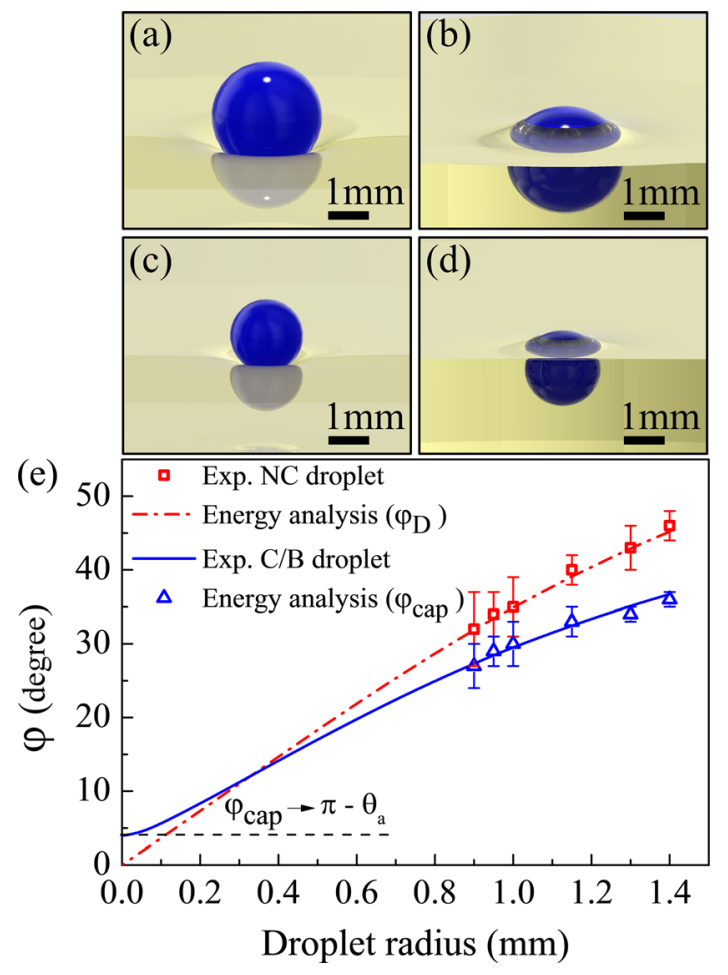

FIG. 4. Energy analysis simulation of both equilibrium states: (a) NC droplet and (b) C/B droplet. (c), (d) The effect of droplet size on the deformation of the free surface. (e) $\varphi_{\mathrm{D}}$ for $\mathrm{NC}$ droplets and $\varphi_{\text {cap }}$ for $\mathrm{C} / \mathrm{B}$ droplets versus droplet radius. found $\left(\varphi_{\mathrm{D}}\right.$ of $35^{\circ}$ for $\mathrm{NC}$ droplet and $\varphi_{\text {cap }}$ of $30^{\circ}$ for $\mathrm{C} / \mathrm{B}$ droplet for a $R_{D}$ of $1 \mathrm{~mm}$ in Figs. S3(a) and S3(b)). ${ }^{19}$

If the droplet kinetic energy is not enough to puncture the oil surface, it will form the NC configuration. Interestingly, the energy analysis could be utilized to estimate the energy barrier that the droplet should overcome to puncture the surface and reach the more stable $\mathrm{C} / \mathrm{B}$ configuration. $\mathrm{E}_{\text {net }}$ divided by droplet weight, $\mathrm{w}_{\mathrm{D}}$, was plotted versus the height of the center of the mass of the droplet from the free surface, $h_{c}$, for $a R_{D}$ of $1 \mathrm{~mm}$ (Fig. 5(a)). The energy reference point was chosen as state $I$ in which the droplet is right on top of the free surface without deforming the oil-air interface. If the droplet has enough kinetic energy it will pass the first equilibrium point (state II) and reach the largest possible surface deflection (state III) where $\varphi_{\mathrm{D}}$ is $90^{\circ}$ (supporting surface tension force, $F_{S}$, is vertical) (Fig. 5(b)). After this point the droplet breaks the oil surface and sinks to state IV in C/B configuration (Fig. 5(c)).

Finally by dissipating the extra energy in a damped oscillation between states IV and VI, it will settle down in the more stable state $\mathrm{V}$. Therefore the energy barrier to achieve $\mathrm{C} / \mathrm{B}$ configuration is the energy difference between state III and state I. The highest release height for which the $\mathrm{NC}$ droplets are formed with a probability of $100 \%, \mathrm{H}_{\mathrm{R}, 1}$, should be equal to $E_{\text {net }}$ of state III divided by $w_{D}$ (Fig. 5(a)). However, by correlating the calculated $\mathrm{H}_{\mathrm{R}, 1}$ with the measured $H_{R, 1}$ for different $R_{D}$ (Fig. $5(d)$ ), it was found that
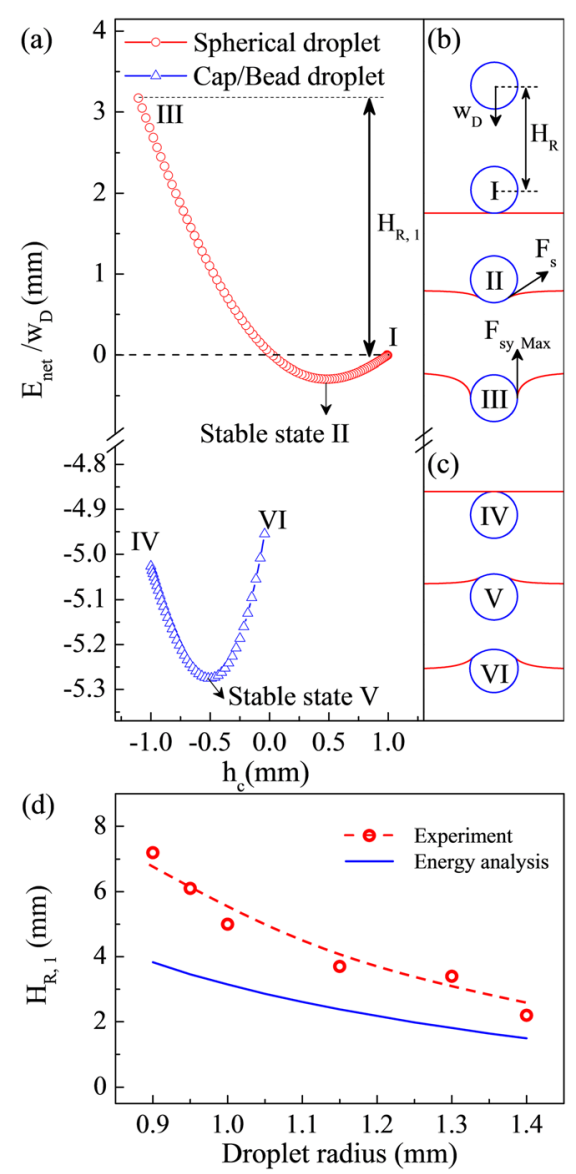

FIG. 5. The transition between the floating droplet's stable equilibrium states with a $R_{D}$ of $1 \mathrm{~mm}$. (a) $E_{\text {net }}$ divided by $w_{D}$ versus $h_{c}$. (b) NC droplet in states I-III. (c) C/B droplet in states IV-VI. (d) $H_{R, 1}$ versus $R_{D}$. 


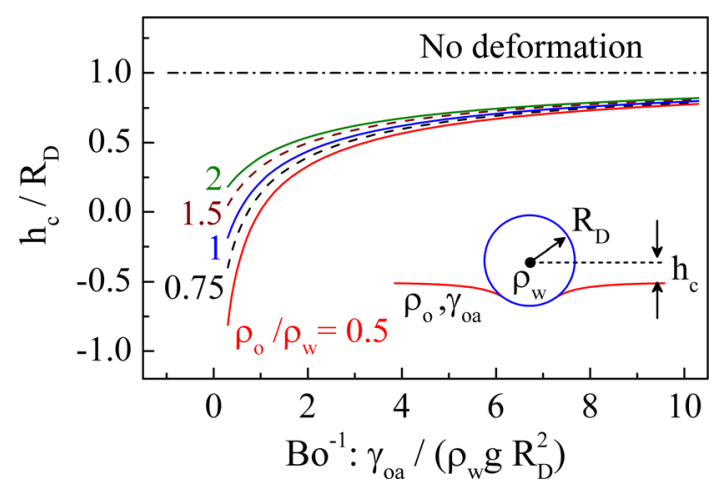

FIG. 6. Oil free surface deformation represented by, $h_{c} / R_{D}$, versus nondimensional parameters obtained from the force balance and energy equations.

consistently a lower value is obtained as compared to the experiment. Since the calculated value is the theoretical upper limit, the higher value obtained in experiment shows that other phenomena resulting in energy dissipation should be considered. ${ }^{15}$ The formation of capillary waves at the oil surface and also the longitudinal waves inside the base medium created by the droplet impact could carry away part of the droplet's kinetic energy. ${ }^{11,16}$

Interestingly, it was found that the thickness of the oil base medium affects $H_{R, 1}$ which not only confirms the creation of waves but also shows that the reflection from the bottom of the container may affect $\mathrm{H}_{\mathrm{R}, 1}$. Therefore in this study a thick layer of oil was used where the reflected wave has no effect on the stability of the droplets.

Further the effect of the droplet size on its stability was studied by calculating $\mathrm{E}_{\text {net }}$ for different $\mathrm{R}_{\mathrm{D}}$ of $\mathrm{NC}$ and $\mathrm{C} / \mathrm{B}$ droplets (Fig. S4). ${ }^{19}$ Based on the force and the energy equations two non-dimensional parameters of $\rho_{\mathrm{o}} / \rho_{\mathrm{w}}$ and the inverse of Bond number, $\mathrm{Bo}^{-1}$, were introduced. The nondimensional, $h_{c} / R_{D}$, is calculated by the energy analysis and plotted versus the two non-dimensional groups of parameters (Fig. 6). This parameter represents the deformation of the free surface, which, as expected, is less for larger $\mathrm{Bo}^{-1}$ and $\rho_{\mathrm{o}} / \rho_{\mathrm{w}}$ (no deformation line in Fig. 6). The data represented by non-dimensional groups of parameters could be used as a guideline to design experiments to form various sizes of floating droplets for the effective droplet manipulation.

This work was supported by NSF (ECCS 1102280), USA and KOSEF (WCU R32-2008-000-10124-0), Korea.

${ }^{1}$ E. F. Greco and R. O. Grigoriev, Phys. Fluids 21, 042105 (2009).

${ }^{2}$ E. Yakhshi-Tafti, H. J. Cho, and R. Kumar, Appl. Phys. Lett. 96, 264101 (2010).

${ }^{3}$ R. Savino, D. Paterna, and M. Lappa, J. Fluid Mech. 479, 307-326 (2003).

${ }^{4}$ A. S. Basu and Y. B. Gianchandani, J. Microelectromech. Syst. 18, 1163-1172 (2009).

${ }^{5}$ Y. Couder, E. Fort, C. H. Gautier, and A. Boudaoud, Phys. Rev. Lett. 94, 177801 (2005).

${ }^{6}$ P. Aussillous and D. Quere, Proc. R. Soc. A: Math. Phys. Eng. Sci. 462, 973-999 (2006).

${ }^{7}$ E. Yakhshi-Tafti, H. J. Cho, and R. Kumar, J. Colloid Interface Sci. 350, 373-376 (2010).

${ }^{8}$ A. Elcrat, R. Neel, and D. Siegel, J. Math. Fluid Mech. 6, 405-429 (2004).

${ }^{9}$ M. S. Khan, D. Kannangara, W. Shen, and G. Garnier, Langmuir 24, 3199-3204 (2008).

${ }^{10}$ O. Reynolds, "On drops floating on the surface of water," Chem. News 44, 211-212 (1881).

${ }^{11}$ G. P. Neitzel and P. Dell'Aversana, Annu. Rev. Fluid Mech. 34, 267-289 (2002).

${ }^{12}$ L. D. Mahajan, Philosophical Magazine Series 7 10, 383-386 (1930).

${ }^{13}$ O. W. Jayaratne and B. J. Mason, Proc. R. Soc. A: Math. Phys. Eng. Sci. 280, 545-565 (1964).

${ }^{14}$ G. E. Charles and S. G. Mason, J. Coll. Sci. 15, 105-122 (1960).

${ }^{15}$ E. M. Honey and H. P. Kavehpour, Phys. Rev. E 73, 027301 (2006).

${ }^{16}$ K. R. Sreenivas, P. K. De, and J. H. Arakeri, J. Fluid Mech. 380, 297-307 (1999).

${ }^{17}$ W. Fox, J. Chem. Phys. 10, 623-628 (1942).

${ }^{18}$ J. S. Rowlinson and B. Wisdom, Molecular Theory of Capillarity (Clarendon Press, Oxford, UK, 1982).

${ }^{19}$ See supplementary material at http://dx.doi.org/10.1063/1.4804242 for calculation of liquid free surface profile; free body diagrams and derivation of force equation; equilibrium states predicted by force and energy analysis; and calculation of net energy for different droplet radii.

${ }^{20}$ C. Huh and L. E. Scriven, J. Colloid Interface Sci. 30, 323-337 (1969).

${ }^{21}$ A. V. Rapacchietta and A. W. Neumann, J. Colloid Interface Sci. 59, 555-567 (1977)

${ }^{22}$ A. V. Rapacchietta, A. W. Neumann, and S. N. Omenyi, J. Colloid Interface Sci. 59, 541-554 (1977). 